\title{
Relief of osteoarthritis with an herbal-amino acid supplement: A randomized double-blind placebo controlled trial
}

\author{
Mark J. S. Miller*, Ross Butler \\ School of Pharmacy \& Medical Sciences, University of South Australia, Adelaide, Australia \\ Email: "markjsm03@gmail.com, Ross.Butler@unisa.edu.au
}

Received 28 June 2012; revised 30 July 2012; accepted 15 August 2012

\begin{abstract}
A redox active medicinal plant and L-leucine mixture (HLM) was investigated in subjects with established osteoarthritis of the knee in a multi-center, randomized, placebo-controlled, double-blind clinical trial. A total of 96 subjects with osteoarthritis were enrolled and randomized to either placebo $(n=38)$ or HLM treatment group $(n=38)$. The HLM group received a combination of Uncaria tomentosa (300 mg), Boswellia serrata (200 mg), Lepidium meyenii (1000 $\mathrm{mg}$ ) and L-Leucine $(700 \mathrm{mg})$ given as 3 capsules once a day. The placebo group received matching capsules with carboxymethylcellulose. The treatment period was 8 weeks, with assessments made at days 7, 14, 28 and 56. The primary outcome was reduction in total WOMAC score. VAS pain, tolerability, investigator assessments, use of rescue medication (acetominophen), and safety assessments of vital signs and laboratory assessments were included. Subject randomization was effective for age, gender and disease severity. In the placebo group $32 / 38$ subjects completed the trial and for HLM 35/38. WOMAC scores (pain, stiffness, physical performance and total) steadily declined over the course of the 8 week study in both groups, but the magnitude was significantly greater for HLM (P < 0.05). Total WOMAC was reduced $\mathbf{4 6 . 5 \%}$ for HLM and $\mathbf{2 5 . 4 \%}$ for placebo. VAS pain was reduced $21.8 \%$ in the placebo group $(p<0.002)$ but the changes were significantly greater $(37.8 \% \mathrm{p}<$ 0.03) with HLM treatment. Investigator's global assessment rating of good-excellent was 24/35 (69\%) for HLM and 14/32 (44\%) for placebo $(P=0.05)$. Rescue medication consumption and tolerability were comparable for HLM and placebo. No safety issues were evident with either group. As expected a placebo effect was observed, nevertheless HLM was clearly more effective in relieving the symptoms of osteoarth-
\end{abstract}

*Corresponding author. ritis. This HLM represents a safe and effective new approach to the management of osteoarthritis symptoms.

Keywords: Osteoarthritis; Dietary Supplement; Cat's Claw; L-Leucine; Boswellia serrata; Maca; Uncaria tomentosa; Lepidium meyenii; Inflammation; Cartilage; Flexibility; Pain; Quality of Life; Stiffness; Arthritis; Nutraceutical; Natural Product

\section{INTRODUCTION}

Osteoarthritis is the most common form of joint disease and reflects a chronic inflammation that over time can result in joint destruction including the loss of articular cartilage and exposure of underlying bone. This condition can cause a significant loss in the quality of life. Current therapeutic interventions are inadequate, largely because they focus on symptomatic relief and do not abrogate the underlying disease process nor stimulate repair processes that may restore joint health for a sustained period [1]. In laboratory studies we have determined that a combination of specific redox-active medicinal plants and the branch-chained amino acid L-leucine (HLM, FlexSure ${ }^{\circledR}$ ) possessed characteristics that were attractive as a potential therapeutic agent for osteoarthritis [2]. HLM was found to activate the genes coding for aggregating cartilage proteoglycan (ACAN) as well as type II collagen (COL2A1), and maintained this heightened expression in the presence of IL- $1 \beta$ which otherwise suppresses these anabolic repair genes. In addition to activating these repair pathways, HLM was able to suppress catabolic pathways (inducible nitric oxide synthase, MMP-9 and MMP-13) and limit IL- $1 \beta$ induced cartilage degradation. Of the three medicinal plants used in the investigative product, Boswellia serrata and Uncaria tomentosa have well described redox-based actions on inflammatory gene expression through the transcripttion factor NF- $k \mathrm{~B}$ [3-6]. On the other hand, the cruci- 
ferous vegetable maca (Lepidium meyenii) has been shown to promote the expression of the repair gene IGF1 in human cartilage cells [7].

These attributes HLM warranted a clinical investigation to further explore its potential as a treatment for osteoarthritis, and is the focus of the present report. The intent of this study was not confirm the gene expression studies noted in isolated cartilage [2], but rather to assess HLM for symptom relief versus placebo in a randomized double-blind controlled trial. In addition to efficacy, the goals were to determine whether HLM was safe using extensive laboratory and vital sign assessments.

\section{METHODS}

\subsection{Clinical Trial Criteria}

This placebo-controlled randomized double-blind multicenter clinical trial was conducted in Mumbai, India under approval of a centralized independent ethics committee ACEAS (Gujarat, India) and registered at clinicaltrials.gov. The conductance of the study was overseen by a contract research organization (Vedic Lifesciences Pvt. Ltd., Mumbai). Four sites were used and subjects were recruited with the following inclusion criteria: age 30 65 of either gender, osteoarthritis diagnosis according to ACR clinical and radiographic criteria, ARA functional class II or III, Kellgren Lawrence radiological severity of Grade II or III of the knee, and maximal visual analog score for pain on movement of between 40 - 80 .

Exclusion criteria include knee pain from conditions other than osteoarthritis, a BMI $>35 \mathrm{~kg} / \mathrm{m}^{2}$, arthroscopy of either knee within the past year, use of analgesics within 7 days of screening, use of corticosteroids or nutraceutical medications for osteoarthritis within the past 3 months. Pregnancy and evidence of major medical conditions, drug dependence and participation in another clinical study within the last 6 weeks were also exclusion criteria.

Withdrawal criteria included serious adverse events that posed serious risk for he subject, development of medical condition that could affect the outcome of the study or any other condition which in the opinion of the investigator justified withdrawal of the subject. Finally, major protocol deviations were grounds for withdrawal. A fixed randomization procedure using an algorithm (Stats direct version 2.70) was used to assign interventions to the participants after participants passed screening procedures and assessments.

\subsection{Treatments}

Placebo or the herbal-leucine mixture (HLM, whose commercial name is FlexSure ${ }^{\circledR}$, Vital g-Netics, Sulfur Springs, Tx) was administered as three capsules delivered once a day, in the absence of meals, for 8 weeks.
HLM consists of a proprietary blend of cat's claw (300 $\mathrm{mg}$, Uncaria tomentosa, Ashancaria $\left.{ }^{\circledR}\right)$, boswellic acid (200 mg, Boswellia serrata), maca (1000 mg, Lepidium meyenii, Macandeum ${ }^{\circledR}$ ) and L-leucine for a total of 2200 mg per day. The L-leucine was pharmaceutical grade manufactured by fermentation and not digested waste proteins. The excipient was magnesium stearate $150 \mathrm{mg}$. Placebo was carboxymethylcellulose (2350 mg).

The primary efficacy variable was total WOMAC score, using a WOMAC scale that was modified for the target population by Chopra [8]. The index consists of 24 questions (5 pain, 2 stiffness, 17 physical function). The Likert scale version of the WOMAC index was used; each question was scored from 0 - 4 with 0 representing none and 4 indicating extreme.

The pain-visual analog score (VAS) was used to assess pain. Patient diaries and consultations with clinic physiccians were used to determine progress at screening, baseline, and then days 7, 14, 38, 56. A central accredited laboratory was used for laboratory biochemical analysis. CRO personnel made monitoring visits every month to ensure that data was collected as per protocol and GCP. An independent statistician reviewed all data and provided statistical assessments. A total of 122 subjects were screened and 76 randomized $(\mathrm{n}=38$ for each placebo and HLM). With 35 subjects completing for HLM and 32 for placebo.

\subsection{Statistical Evaluation}

Statistical evaluation was performed by an independent statistician who was contracted by the CRO, Vedic Lifesciences, Pty., Ltd. Efficacy was assessed using "intention-to-treat" (ITT) data where appropriate, using ANOVA and a repeated measures test where appropriate. Investigator assessments summarized in contingency tables was assessed using the Pearson's Chi-Square test. The statistical software program Stats Direct 2.70 was used. Significance was taken a probability at the $5 \%$ level or less.

\section{RESULTS}

\subsection{Subject Demographics}

Subject demographics enrolled in the clinical trial are summarized in Table 1. Randomization was effective with characteristics being indistinguishable between the two groups. Baseline efficacy metrics were comparable in the two groups, Table 2. Compliance was comparable between groups and averaged $>96 \%$.

\subsection{Total WOMAC_Primary Efficacy Variable}

Osteoarthritis is well known to be sensitive to a placebo effect and this was evident in this trial with a steady re- 
Table 1. Subject demographics.

\begin{tabular}{ccc}
\hline Demographic Characteristics & Placebo & HLM \\
\hline Age (years) & $53.1 \pm 8.9$ & $54.1 \pm 7.7$ \\
Gender (F:M) & $18: 20$ & $24: 14$ \\
BMI & $26.5 \pm 3.3$ & $26.5 \pm 3.1$ \\
$\begin{array}{c}\text { ARA Functional Class } \\
\text { (Class II: Class III) }\end{array}$ & $34: 4$ & $33: 5$ \\
$\begin{array}{c}\text { Kellgren Lawrence criteria } \\
\text { (Grade 2: Grade 3) }\end{array}$ & $29: 9$ & $26: 12$ \\
\hline
\end{tabular}

Results expressed as mean \pm SD. There was no statistical difference between groups.

Table 2. Baseline efficacy variables.

\begin{tabular}{lcc}
\hline Baseline Values & Placebo $(\mathrm{n}=38)$ & HLM $(\mathrm{n}=38)$ \\
\hline WOMAC-Pain & $6.6 \pm 3.4$ & $7.3 \pm 3.3$ \\
WOMAC-Stiffness & $2.8 \pm 1.8$ & $2.8 \pm 1.5$ \\
WOMAC-Physical Function & $25.4 \pm 11.4$ & 29. \\
WOMAC Total & $34.8 \pm 15.8$ & $39.3 \pm 13.4$ \\
VAS Pain & $63.2 \pm 10.0$ & $59.5 \pm 10.0$ \\
\hline
\end{tabular}

Results expressed as mean \pm SD. There was no statistical difference between groups.

duction in total WOMAC with placebo treatment over the 56 day trial period. However, reductions in total WOMAC were significantly greater with HLM treatment. This was noted from day 14 through to day 56 (Figure 1) with HLM. On a percentage basis HLM resulted in a $46.5 \%$ in total WOMAC, with only $25.4 \%$ with placebo treatment at day 56.

\subsection{WOMAC - Pain, Stiffness and Physical Function}

Similar to Total WOMAC, assessments for pain, stiffness and physical function indicated that HLM was significantly more effective than placebo. These results are summarized in Figures 2-4. HLM resulted in signifycantly greater improvements than placebo for all metrics $(\mathrm{p}<0.05)$. Both placebo and HLM showed a time-dependent improvement although this was more pronounced with HLM.

\subsection{VAS Pain}

Pain, assessed with a visual analog scale, was indistinguishable at baseline between groups: placebo $59.7 \pm 2.0$, HLM $59.9 \pm 1.7$. VAS pain was reduced with both placebo and HLM treatments (21.8 vs. $37.8 \%, \mathrm{p}<0.002)$ over the 8 week study period. However, the benefits were significantly greater with HLM (Figure $5, \mathrm{p}<0.05$ ).

\subsection{Investigator's Global Assessment of Efficacy}

Categorization of the global assessment of efficacy used

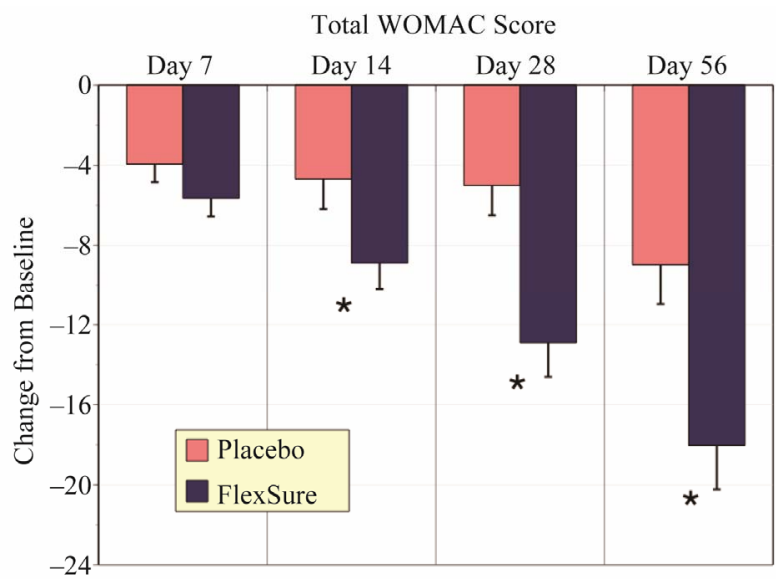

Figure 1. Changes in total WOMAC score. Results expressed as change from baseline (mean \pm sem). The $*$ signifies a statistical difference from placebo of at least $\mathrm{p}<0.05$.

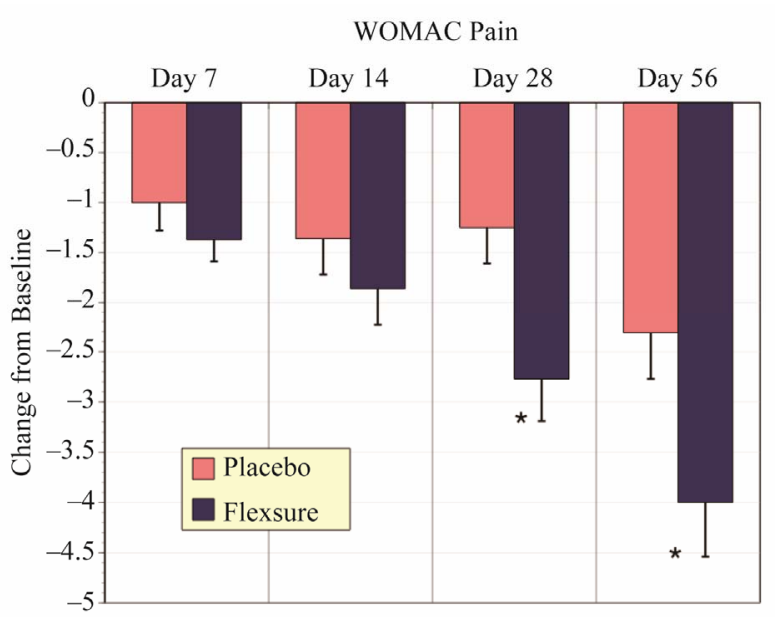

Figure 2. Changes in WOMAC pain scores. Results expressed as change from baseline (mean \pm sem). The $*$ denotes a statistical difference from placebo.

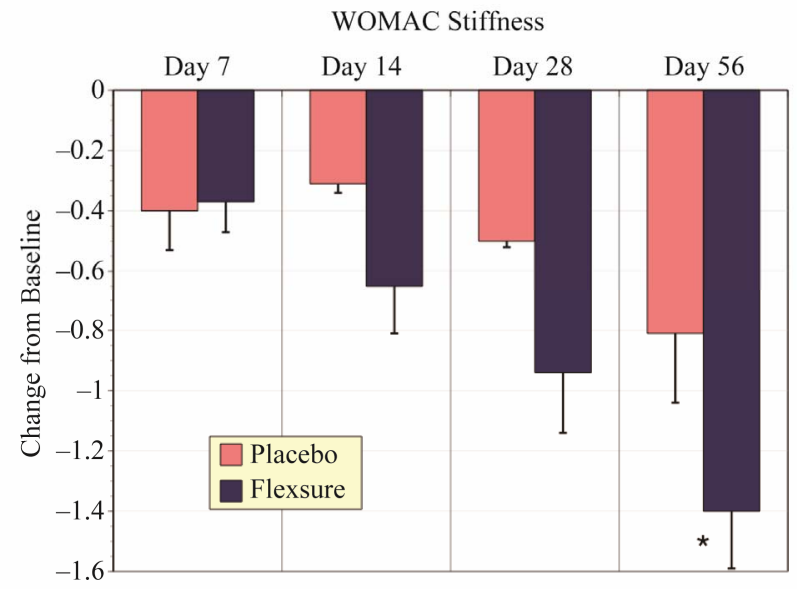

Figure 3. Changes in WOMAC stiffness scores. Results depict the change from baseline (mean \pm sem). The $*$ represents a statistical difference from placebo of at least $\mathrm{p}<0.05$. 


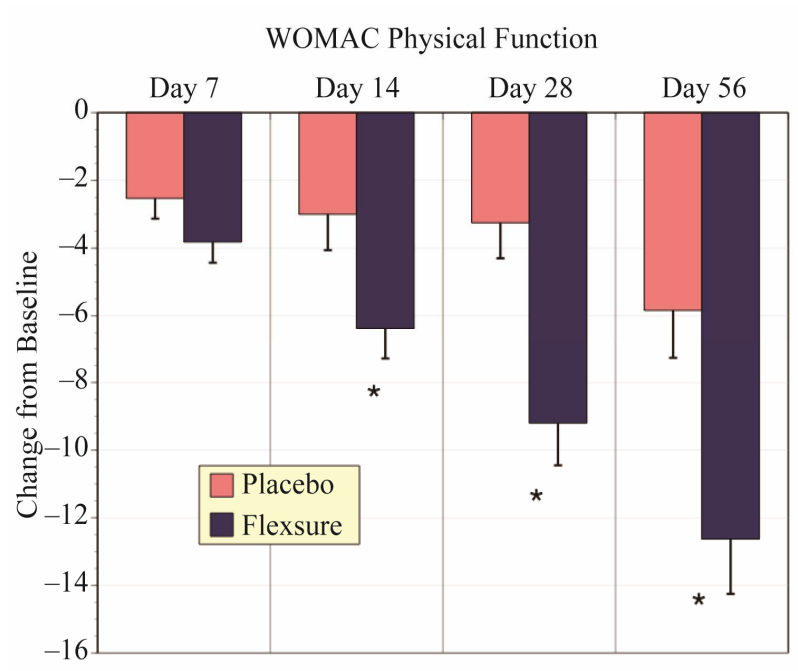

Figure 4. Changes in WOMAC physical function scores. Results depict the changes from baseline (mean \pm sem). The * designates a statistically significant difference from placebo of at least $\mathrm{p}<0.05$.

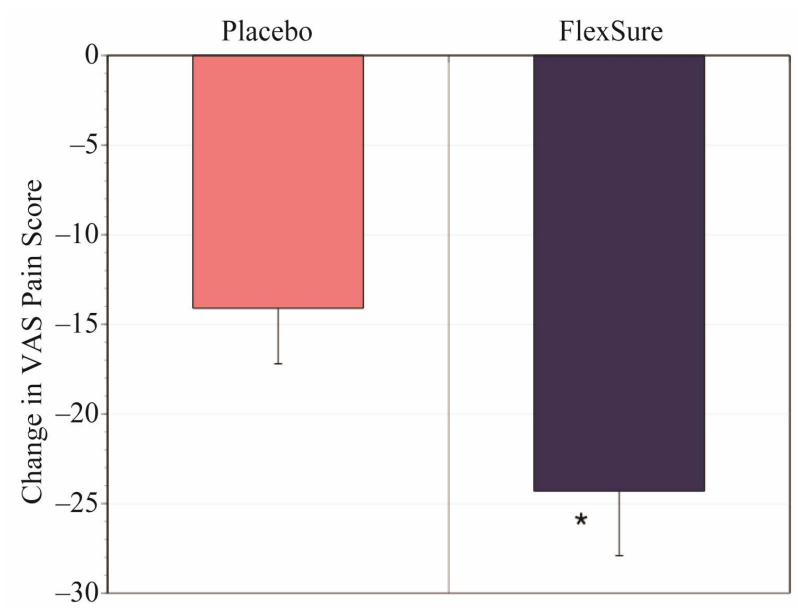

Figure 5. Change in VAS pain. Results depict the change in VAS pain score after 56 days as noted from baseline (mean \pm sem). The $*$ denotes a statistical significant difference from placebo $(\mathrm{p}<0.05)$.

4 categories-poor, fair, good and excellent. Combining the groups poor/fair and good/excellent HLM was marginally more effective than placebo (Table 3, $\mathrm{p}=0.05$ ). HLM treatment had a $68.6 \%$ assessment of good-excellent in contrast to $43.8 \%$ for placebo.

\subsection{Consumption of Rescue Medication}

In accord with ethics committee directions the subjects had free access to the rescue medication, acetominophen. Consumption of the rescue medication was determined at each time period (days 7, 14, 28, 56. No difference was determined between placebo and HLM treatments (Table 4).
Table 3. Investigator's global assessment.

\begin{tabular}{ccc}
\hline Categorization of Ratings & Placebo $(\mathrm{n}=32)$ & HLM $(\mathrm{n}=35)$ \\
\hline Excellent \& Good & 14 & 24 \\
Fair \& Poor & 18 & 11 \\
\hline
\end{tabular}

$\mathrm{p}=0.05$ Chi Square test placebo vs. HLM.

Table 4. Rescue medication consumption.

\begin{tabular}{ccc}
\hline $\begin{array}{c}\text { Rescue Medication } \\
\text { Consumption }\end{array}$ & Placebo & HLM \\
\hline Days 0 - 7 & 0 & $1.4 \pm 8.4$ \\
Days 7 - 14 & $1.9 \pm 3.1$ & $1.2 \pm 2.3$ \\
Days 14 - 28 & $2.7 \pm 3.6$ & $2.3 \pm 3.7$ \\
Days 28 - 56 & $5.2 \pm 8.2$ & $4.4 \pm 6.9$ \\
\hline
\end{tabular}

Results expressed as mean \pm SD. There was no statistical difference between groups.

\subsection{Responsiveness and Onset of Action}

Exceeding a $20 \%$ change in symptom assessments is a commonly used metric for determining responsiveness and onset of action. The HLM group displayed an average reduction of pain (24\%) and stiffness (22\%) within 7 days and all assessments (pain, stiffness, physical function and total WOMAC) by day 14. By contrast placebo did not achieve a $20 \%$ reduction in total WOMAC until the conclusion of the study (day 56). These results indicate that HLM displayed a significantly greater magnitude of benefits with an earlier onset of action than placebo.

\subsection{Safety Assessments}

Safety assessments were comprehensive and included laboratory and measurements of vital signs. In terms of laboratory assessments there was no difference in the values noted with placebo or HLM for total white blood cells, RBC, hemaglobin, hematocrit, platelets, differenttial white blood cell counts, erythrocyte sedimentation rate, serum creatine, SGPT, and fasting blood sugar (Table 5). Vital sign assessments were also not distinguishable between placebo and HLM (Table 6). This includes pulse rate, systolic blood pressure and diastolic blood pressure.

There were 6 adverse events reported during the study. Of these 5 were of the mild to moderate category, and one was severe. There was a rise in serum SGPT in 2 subjects, one each in the placebo and HLM group. A rise in fasting blood sugar level was noted in 2 subjects in the placebo arm. There was a single report of nausea and vomiting in both the placebo and HLM group. Tolerability rating for HLM vs. placebo was fair in 3 versus 1 , and good in 32 vs. 31. 
Table 5. Laboratory safety assessments.

\begin{tabular}{lcccc}
\hline Variable & Placebo Baseline & $\begin{array}{c}\text { Placebo } \\
\text { End of treatment }\end{array}$ & $\begin{array}{c}\text { HLM } \\
\text { Baseline }\end{array}$ & $\begin{array}{c}\text { HLM } \\
\text { End of treatment }\end{array}$ \\
\hline WBC $\times 10^{3}$ /cu.mm & $6.5 \pm 1.3$ & $6.7 \pm 1.7$ & $7.1 \pm 1.6$ & $7.3 \pm 1.8$ \\
RBC million/cu.mm & $4.8 \pm 0.6$ & $4.8 \pm 0.6$ & $4.7 \pm 0.6$ & $4.6 \pm 0.7$ \\
Hb gm/dl & $13.5 \pm 1.5$ & $13.5 \pm 1.6$ & $13.2 \pm 1.7$ & $13.2 \pm 1.8$ \\
Hematocrit \% & $40.9 \pm 4.3$ & $40.6 \pm 4.4$ & $40.5 \pm 5.0$ & $40.1 \pm 4.8$ \\
Platelet 1000/cu.mm & $258.6 \pm 53.9$ & $254.8 \pm 65.7$ & $265.4 \pm 60.1$ & $274.3 \pm 73.5$ \\
Neutrophil \% & $56.8 \pm 7.9$ & $59.4 \pm 9.2$ & $60.0 \pm 10.3$ & $63.9 \pm 9.7$ \\
Lymphocyte \% & $32.9 \pm 7.5$ & $31.0 \pm 8.5$ & $30.5 \pm 9.0$ & $27.7 \pm 7.6$ \\
Eosinophil \% & $4.0 \pm 3.4$ & $4.7 \pm 4.0$ & $3.6 \pm 4.3$ & $3.6 \pm 4.1$ \\
Basophil \% & 0 & 0 & 0 & 0 \\
Monocyte \% & $6.2 \pm 2.0$ & $4.9 \pm 2.2$ & $5.9 \pm 2.4$ & $4.8 \pm 2.0$ \\
ESR & $15.0 \pm 7.7$ & $19.1 \pm 15.7$ & $17.9 \pm 8.6$ & $19.4 \pm 11.0$ \\
Serum Creatine mg/dl & $0.79 \pm 0.17$ & $0.86 \pm 0.17$ & $0.84 \pm 0.18$ & $0.91 \pm 0.23$ \\
SGPT U/L & $39.7 \pm 8.1$ & $42.9 \pm 14.2$ & $37.7 \pm 10.6$ & $43.2 \pm 15.2$ \\
Fasting Blood Sugar mg/dl & $95.0 \pm 14.9$ & $105.2 \pm 34.1$ & $95.8 \pm 13.4$ & $98.4 \pm 17.2$ \\
\hline
\end{tabular}

Results expressed as mean \pm SD. There were no statistical differences between groups.

Table 6. Vital signs.

\begin{tabular}{|c|c|c|c|c|}
\hline Variables & Placebo Baseline & $\begin{array}{c}\text { Placebo } \\
\text { End of treatment }\end{array}$ & $\begin{array}{c}\text { HLM } \\
\text { Baseline }\end{array}$ & $\begin{array}{c}\text { HLM } \\
\text { End of treatment }\end{array}$ \\
\hline Pulse & $74.7 \pm 6.2$ & $75.3 \pm 6.2$ & $74.6 \pm 5.7$ & $75.4 \pm 6.4$ \\
\hline Systolic BP mm HG & $126.8 \pm 11.3$ & $128.7 \pm 11.9$ & $127.8 \pm 12.1$ & $127.0 \pm 9.9$ \\
\hline Diastolic BP mm HG & $81.8 \pm 6.3$ & $83.3 \pm 7.4$ & $82.5 \pm 7.1$ & $81.9 \pm 7.4$ \\
\hline
\end{tabular}

Results expressed as mean \pm SD. There was no statistical difference between groups.

\section{DISCUSSION}

Osteoarthritis compromises the quality of life and is remarkably common condition with $12.1 \%$ of the US population aged 25 - 74 having osteoarthritis of at least one joint [9]. Age is a major risk factor and the limitations on physical performance and concomitant pain results in osteoarthritis being a major societal burden. Despite this burden and the high incidence osteoarthritis there has been little in the way of treatments that modify the disease course and alleviate symptoms. The current standard treatment is NSAIDs, which alleviate symptoms like pain but do not alter the course of cartilage loss and joint destruction. In addition, NSAIDs are associated with a high rate of gastrointestinal, cardiovascular and renal side-effects, which can be fatal [10].

Innovations that provide symptomatic relief as well as alter the course of the disease are truly needed and as an alternative to pharmaceutical approaches there has been considerable interest in the nutraceutical approaches. Ingested glucosamine, which is a structural element of cartilage, has been one of the more popular and widely studied [9]. However, despite early encouragement recent studies suggest that there is little benefit to gluco- samine over placebo. A major reason for the poor responsiveness to glucosamine, or its related more complicated polymer chondroitin, is the disconnect between ingestion and the insertion of these matrix elements into joint matrix.

Bioavailability at the joint level is one limitation. Chondroitin is poorly digested and ingested glucosamine is cleared by the liver; breaking down to its glucose and amine components [9]. A further concern is that this glucose burden may pose issues for diabetics and prediabetics $[11,12]$. Another problem that limits joint repair is that ongoing inflammation suppresses the genes responsible for repairing cartilage $[1,2]$. In other words, cartilage repair is limited in arthritis through the combined effects of activated catabolic processes and suppressed anabolic, repair mechanisms. Ingesting additional matrix substrate, like type II collagen or glucosamine, under these circumstances without an active process that inserts the substrate into the cartilage matrix, is an inherently inefficient process. For this reason clinical results observed with either glucosamine or hydrolyzed collagen supplements have yielded mixed results.

The test agent used in this clinical trial, the combina- 
tion of medicinal plants and leucine (HLM, FlexSure ${ }^{\circledR}$ ) has an entirely different approach to the problem and possesses attractive disease modifying agent properties. In human cartilage explants and chondrocyte culture, HLM demonstrated unique attributes that limited the processes that cause cartilage breakdown while at the same time activating the genetic resources that determine cartilage repair [2]. This research was performed in human cartilage explants and cultured human chondrocytes treated with IL- $1 \beta$ to mimic inflammation. HLM was effective in suppressing major, catabolic processes in human cartilage, specifically, expression of inducible nitric oxide synthase, MMP-9 and MMP-13 [2]. The master protein switch controlling inflammation associated gene expression, NF-kB, was suppressed by HLM $[2,13]$. This confirms what we and others have previously noted with the individual medicinal plant components [3-7,14]. Concomitantly, the release of glucosamine from the cartilage matrix was blocked by HLM despite the ongoing presence of IL- $1 \beta$.

While HLM prevented cartilage breakdown by suppression of redox-sensitive gene activation, it was also able to promote the gene expression of critical repair genes-ACAN and COL2A1 [2]. In other words despite the presence of IL- $1 \beta$, HLM was able to transform the chondrocyte gene expression profile from catabolic to anabolic and repair focused. The role of L-leucine has not been fully explored in human cartilage and joints. It is a known anabolic factor in skeletal muscle [15,16], and recently it was reported that L-leucine was consumed at an excessive rate as a metabolic fuel by inflamed human synovium [17], suggesting that joint inflammation has a central component that disturbed leucine actions and/or metabolism. However, the individual contribution of L-leucine to the benefits of HLM is unknown.

The benefits noted in those in vitro studies on human cartilage are affirmed and extended to therapeutic applications in osteoarthritis in this study. Of critical importance HLM was demonstrated to be safe. There were no differences in vital signs or laboratory-based safety assessments between HLM and placebo (Tables 5-6).

Efficacy in alleviating the symptoms of osteoarthritis was assessed using the WOMAC score, which measures pain, flexibility/stiffness and physical performance with the summary of all of these measurements becoming the Total WOMAC score. VAS pain was also used as an assessment of efficacy. For all of these metrics, HLM was significantly more effective than placebo. Osteoarthritis, like other painful conditions, has a well-described placebo effect [18]. This placebo effect can mask effective agents because of the high background noise, and large numbers of subjects is sometimes used to help distinguish efficacy of test agents from placebo. Rescue medi- cation use is an example of another potential masking factor. However, the use of acetominophen as a rescue medication was comparable between placebo and HLM.

However, in this study it is very clear that the benefits of HLM were readily distinguishable from placebo and this was apparent very early in the clinical trial (Figures 1-5). Significant improvements in pain relief, stiffness and mobility and physical performance and comfort were noted with HLM. Blinded global assessments of the interventions (HLM vs. placebo) indicated that these benefits to the study investigators and co-ordinators (Table 3). This is not to state that a placebo effect was absent in this study. By contrast it was very evident, with placebo improving all metrics related to efficacy. However, in all cases HLM produced a response whose magnitude was significantly better than placebo.

By following the progress of benefits throughout the course of the 2 month study we are able to provide additional evidence distinguishing HLM from placebo. These time-related assessments also indicate that the ability to establish a benefit of HLM over placebo begins at 7 days and is emphatic after 14 days of consumption. Previous clinical trials on glucosamine and related matrix-based nutraceuticals suggest an onset of action of months. The earlier onset of action of HLM compared to matrix-based interventions is most likely explained by the combined actions of limiting ongoing inflammation initiated catabolism while optimizing repair mechanisms with HLM [2-7,14-16].

\section{CONCLUSION}

An investigational agent consisting of a combination of redox-active medicinal plants and the branch-chained amino acid L-leucine, was effective in comprehensively alleviating arthritis symptoms in subjects with osteoarthritis of the knee. HLM should be considered a safe and effective approach in the treatment of osteoarthritis.

\section{ACKNOWLEDGEMENTS}

This clinical trial was performed the assistance of the contract research organization, Vedic Lifescience, Pty. Ltd., and was sponsored by Vital g-Netics, LLC.

Neither author has a conflict of interest; there is no fiscal relationship with the manufacturer of FlexSure ${ }^{\circledR}$ and compensation for the conductance of this research project nor the development of this manuscript. Both authors contributed to the study design, analysis and manuscript preparation.

\section{REFERENCES}

[1] Bay-Jensen, A.C., Hoegh-Madsen, S., Dam, E., Henriksen, K., Sondergaard, B.C., Pastoureau, P., Qvist, P. and Karsdal, M.A. (2010) Which elements are involved in 
reversible and irreversible cartilage damage in osteoarthritis. Rheumatology International, 30, 435-442. doi:10.1007/s00296-009-1183-1

[2] Miller, M.J.S., Akhtar, N. and Haqqi, T.M. (2011) Effect of a herbal-leucine mix on the IL- $1 \beta$-induced cartilage degradation and inflammatory gene expression in human chondrocytes. BMC Complementary and Alternative Medicine, 11, 66. doi:10.1186/1472-6882-11-66

[3] Kimmatkar, N., Thawani, V., Hingorani, L. and Khiyani, R. (2003) Efficacy and tolerability of Boswellia serrata extract in treatment of osteoarthritis of knee-A randomized double blind placebo controlled trial. Phytomedicine, 10, 3-7. doi:10.1078/094471103321648593

[4] Sontakke, S., Thawani, V., Pimpalkhute, S., Kabra, P., Babhulkar, S. and Hingorani, L. (2007) Open, randomized, controlled clinical trial of Boswellia serrata extract as compared to valdecoxib in osteoarthritis of knee. Indian Journal of Pharmacology, 39, 27-29. doi:10.4103/0253-7613.30759

[5] Sandoval-Chacon, M., Thompson, J.H., Liu, X., Mannick, E.E., Sadowska-Krowicka, H., Charbonnet, R., Clark, D.A. and Miller, M.J.S. (1998) Anti-inflammatory actions

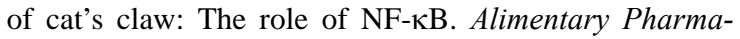
cology and Therapeutics, 12, 1279-1289. doi:10.1046/j.1365-2036.1998.00424.x

[6] Sandoval, M., Charbonnet, R.M., Okuhama, N.N., Roberts, J., Krenova, Z., Trentacosti, A.M. and Miller, M.J.S. (2000) Cat's claw inhibits TNF production and scavenges free radicals: Role in cytoprotection. Free Radical Biology and Medicine, 29, 71-78. doi:10.1016/S0891-5849(00)00327-0

[7] Miller, M.J., Ahmed, S., Bobrowski, P. and Haqqi, T.M. (2006) The chrondroprotective actions of a natural product are associated with the activation of IGF-1 production by human chondrocytes despite the presence of IL-1beta. BMC Complementary and Alternative Medicine, 6, 13. doi:10.1186/1472-6882-6-13

[8] Chopra, A. (2004) Rheumatology: Made in India (Camps, COPCORD, HLA, Ayurveda, HAQ, WOMAC and Drug Trials). Journal of Indian Rheumatology Association, 12, 43-53.

[9] Clegg, D.O., et al. (2006) Glucosamine, chondroitin sulfate, and the two in combination for painful knee osteoarthritis. New England Journal of Medicine, 354, 795808. doi:10.1056/NEJMoa052771
[10] Bjarnason, I. and Thjodleifsson, B. (1999) Gastrointestinal toxicity of non-steroidal anti-inflammatory drugs: The effect of nimesulide compared with naproxen on the human gastrointestinal tract. Rheumatology, 38, 24-32. doi:10.1093/rheumatology/38.suppl_1.24

[11] Biggee, B.A., Blinn, C.H., Nuity, M., Silbert, J.E. and McAlindon, T.E. (2007) Effects of oral glucosamine sulfate on serum glucose and insulin during an oral glucose tolerance test of subjects with osteoarthritis. Annals of the Rheumatic Diseases, 66, 260-262. doi:10.1136/ard.2006.058222

[12] Pham, T., Cornea, A., Blick, K.E., Jenkins, A. and Scofield, R.H. (2007) Oral glucosamine in doses used to treat osteoarthritis worsens insulin resistance. American Journal of the Medical Sciences, 333, 333-339. doi:10.1097/MAJ.0b013e318065bdbe

[13] Roman-Blas, J.A. and Jimenez, S.A. (2006) NF-kappaB as a potential therapeutic target in osteoarthritis and rheumatoid arthritis. Osteoarthritis Cartilage, 14, 839848. doi:10.1016/j.joca.2006.04.008

[14] Piscoya, J., Rodriguez, Z., Bustamante, S., Miller, M.J.S. and Sandoval, M. (2001) Efficacy and safety of freeze dried cat's claw in osteoarthritis of the knee: Mechanisms of action of the specie Uncaria guianensis. Inflammation Research, 50, 442-448. doi:10.1007/PL00000268

[15] Crowe, M.J., Weatherson, J.N. and Bowden, B.F. (2006) Effects of dietary leucine supplementation on exercise performance. European Journal of Applied Physiology, 97, 664-672. doi:10.1007/s00421-005-0036-1

[16] Li, F., Yin, Y., Tan, B., Kong, X. and Wu, G. (2001) Leucine nutrition in animals and humans: mTOR signaling and beyond. Amino Acids, 41, 1185-1193. doi:10.1007/s00726-011-0983-2

[17] Adams, S.B. Jr., Setton, L.A., Kensicki, E., Bolognesi, M.P., Toth, A.P. and Nettles, D.L. (2012) Global metabolic profiling of human osteoarthritic synovium. Osteoarthritis and Cartilage, 20, 64-67. doi:10.1016/j.joca.2011.10.010

[18] Zhang, W., Robertson, J., Jones, A.C., Dieppe, P.A. and Doherty, M.A. (2008) Placebo effect and its determinants in osteoarthritis: Meta-analysis of randomized controlled trials. Annals of the Rheumatic Diseases, 67, 1716-1723. doi:10.1136/ard.2008.092015 